\title{
I 27 Coronary Wall MRI detects subclinical atherosclerosis in asymptomatic individuals in the multi-ethnic study of atherosclerosis (MESA)
}

\author{
Robson Macedo*1, Shaoguang Chen ${ }^{1}$, Shenghan Lai ${ }^{1}$, Steven Shea ${ }^{2}$, \\ Ashkan A Malayeri ${ }^{1}$, Moyses Szklo ${ }^{3}$, Joao AC Lima ${ }^{1}$ and David A Bluemke ${ }^{1}$
}

\author{
Address: ${ }^{1}$ Johns Hopkins University, School Of Medicine, Baltimore, USA, ${ }^{2}$ Siemens Medical Systems, Baltimore, USA and ${ }^{3}$ Johns Hopkins \\ University, |School Of Public Health, Baltimore, USA \\ * Corresponding author
}

from I I th Annual SCMR Scientific Sessions

Los Angeles, CA, USA. I-3 February 2008

Published: 22 October 2008

Journal of Cardiovascular Magnetic Resonance 2008, 10 (SuppI I):A28 doi:10.1 I86/1532-429X-10-SI-A28

This abstract is available from: http://jcmr-online.com/content/I0/SI/A28

(C) 2008 Macedo et al; licensee BioMed Central Ltd.

\section{Introduction}

Coronary wall MRI has been shown to demonstrate increased thickness of the coronary wall in individuals with documented coronary artery disease.

\section{Purpose}

The purpose of this study was to evaluate the usefulness of coronary wall MRI as a measure of atherosclerotic disease burden in an asymptomatic population free of clinical cardiovascular disease.

\section{Methods}

Forty eight consecutive subjects in the Multi-Ethnic Study of Atherosclerosis (MESA) study underwent coronary wall MRI of the left and right coronary arteries at $5 \mathrm{~mm}$ intervals using a black blood turbo spin echo technique.

\section{Results}

Maximum coronary wall thickness was greater for subjects with 2 or more cardiovascular risk factors than for those with 1 or no risk factors $(2.59 \pm 0.33 \mathrm{~mm}$ versus $2.36 \pm$ $0.30 \mathrm{~mm}$, respectively, $\mathrm{p}=0.05$.) For subjects with zero coronary calcium score, the mean and maximum coronary wall thickness for subjects with 2 or more risk factors for coronary artery disease were greater than the wall thickness for subjects with 1 or no risk factors (mean thickness: $1.95 \pm 0.17 \mathrm{~mm}$ versus $1.7 \pm 0.19 \mathrm{~mm}$; maximum thickness: $2.67 \pm 0.24 \mathrm{~mm}$ versus $2.32 \pm 0.27 \mathrm{~mm}$, respectively, $\mathrm{p}<0.05)$. Subjects with carotid intimalmedial thickness (IMT) greater than one standard deviation (SD) above the mean had significantly higher mean and maximum coronary wall thickness compared to subjects with carotid IMT less than or equal to one SD above the mean $(\mathrm{p}<0.05)$.

\section{Conclusion}

MRI detects increased coronary wall thickness in asymptomatic individuals with subclinical markers of atherosclerotic disease and in individuals with zero coronary calcium score. 


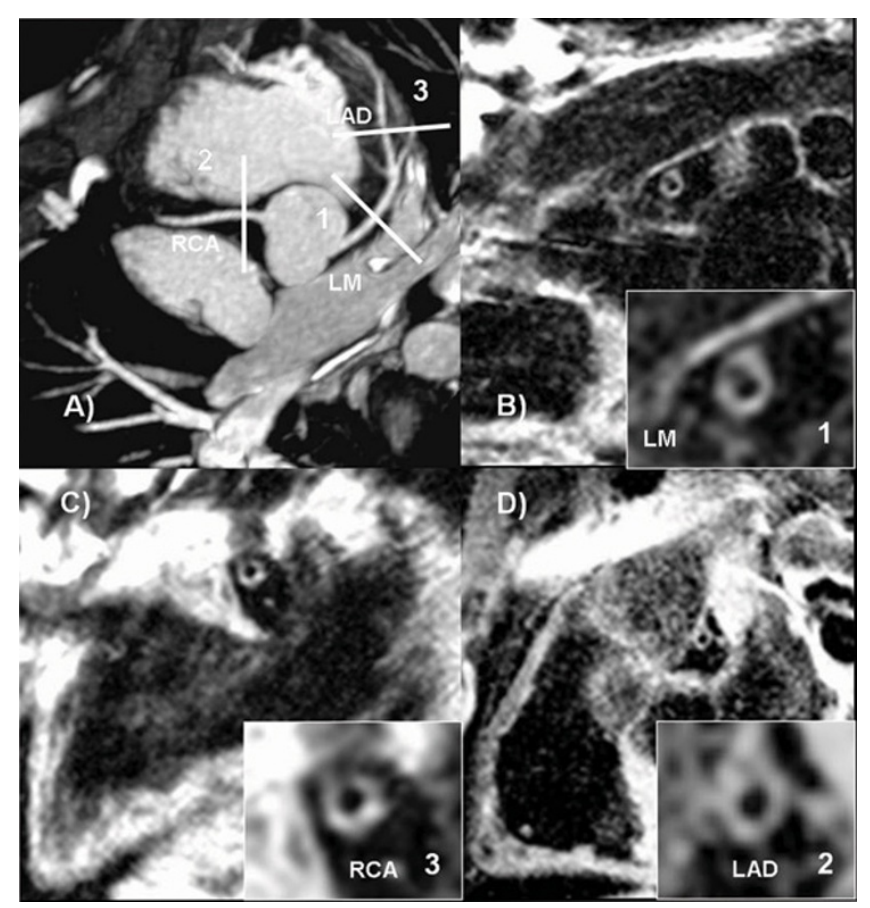

Figure I

Normal coronary MR angiogram with increased coronary artery wall thickness. 73 year-old male participant with 2 risk factors for CAD and Agatston score of 200. Coronary wall MRI was used to measure of atherosclerotic disease burden in individuals without clinical cardiovascular disease. MRI detected increased coronary wall thickness in individuals with subclinical markers of atherosclerotic disease and in individuals with zero coronary calcium score. A) Coronary MRA demonstrating slice positioning of representative slices for coronary wall imaging. $B$ ), C) and D) Coronary wall images of the LM, RCA and LAD, respectively, showing increased wall thickness. 